\title{
Queratoconjuntivitis por adenovirus generadas a partir de una consulta oftalmológica
}

\author{
Pablo Romero C., Marlene Vogel G., Federica Solanes y Vivian Luchsinger F.
}

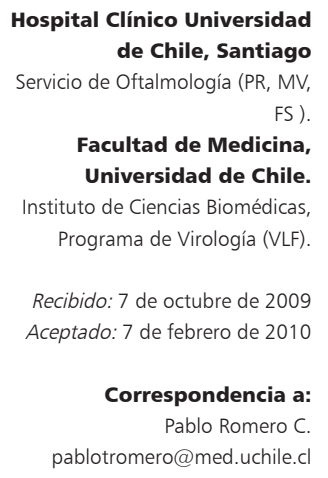

Hospital Clínico Universidad

Facultad de Medicina, uto de Ciencias Biomédicas, Programa de Virología (VLF)

Pablo Romero C.

\begin{abstract}
Adenovirus keratoconjunctivitis acquired in an ophtalmology clinic
Introduction: Eye infection is a common cause of ophtalmologic consultation. Adenovirus keratoconjunctivitis outbreaks are common worldwide but its impact and clinical characteristic in Chilean population is unkown. Objective: To describe a series of adenovirus keratoconjunctivitis cases. Patients and Method: The index case and contacts received medical care in the Hospital Clínico Universidad de Chile between April and August 2006. A complete ophthalmologic exam and microbiologic evaluation was performed. Results: Nine patients presented a pattern of characteristic epidemic keratoconjunctivitis. In x cases sub-corneal epithelial infiltrates were observed for a period of more than six months. Three affected patients were ophtalmologists, staff at the Hospital. In seven patients ADV was isolated all bellonging to type D genus. Conclusions: Adenovirus type D caused epidemic keratoconjunctivitis in a series of Chilean individuals. Ophthalmologist may have transmitted the virus to patients.
\end{abstract}

Key words: Epidemic keratoconjunctivitis, adenovirus.

Palabras clave: Queratoconjuntivitis epidémica, adenovirus.

\section{Introducción}

$\mathrm{L}$ a patología ocular infecciosa es una entidad clínica muy frecuente, constituyendo la conjuntivitis y queratoconjuntivitis de este origen el $70 \%$ de la consulta oftalmológica en el nivel primario en países desarrollados ${ }^{1,2}$, situación que parece ser similar en países en vías de desarrollo, como el nuestro.

Los brotes de queratoconjuntivitis epidémica (QCE), principalmente causados por adenovirus (ADV), están ampliamente descritos en la literatura, tanto en Europa como en Estados Unidos de Norteamérica ${ }^{2,3}$. La mayoría de ellos presenta una fuente de infección común que puede ser una consulta oftalmológíca ${ }^{4-8}$ o una piscina ${ }^{9}$. Se producen además casos secundarios en el entorno familiar, social y de ambientes de trabajo por transmisión directa o indirecta ${ }^{10}$. Un pequeño porcentaje de pacientes no refiere alguna de las fuentes de infección descritas no pudiéndose determinar su origen.

Los ADV son importantes agentes de pandemias y epidemias de queratoconjuntivitis en el extranjero ${ }^{5-10}$; sin embargo, desconocemos la ocurrencia y el impacto de ellas en nuestro país. Se encuentran ampliamente distribuidos en seres humanos y otros animales, siendo especie específicos ${ }^{11,12}$. Pertenecen al género Mastadeno- virus de la familia Adenoviridae ${ }^{11}$. Estos virus son estables frente a agentes químicos o físicos como el calor y $\mathrm{pH}$, sobreviviendo largo tiempo en el ambiente. Se transmiten con facilidad mediante gotitas (gotas mayores a $5 \mu \mathrm{m}$ ) y por vía fecal oral y, menos frecuentemente, por aerosoles respiratorios ${ }^{14}$.

Se describen seis géneros de ADV - A a F-, 33\% de los cuales se asocian a enfermedades en los seres huma$\operatorname{nos}^{13,14}$. Los ADV del género $\mathrm{F}$ producen principalmente infecciones respiratorias, los $\mathrm{B}$ y $\mathrm{C}$, gastroenteritis y los $\mathrm{B}$ y D conjuntivitis ${ }^{15-17}$. Además de infección respiratoria, gastrointestinal, genitourinaria, ocular y dermatológica, los ADV pueden afectar, aunque con menor frecuencia, el corazón y a los sistemas nervioso central, auditivo y endocrino $^{18,19}$.

Algunos de los cuadros oftalmológicos que puede producir el ADV son la conjuntivitis folicular aguda inespecífica, la fiebre faringo-conjuntival y la QCE. Esta última está asociada con frecuencia a los serotipos 8, 19 y 37 de $\mathrm{ADV}^{21}$.

\section{Objetivos}

Describir una serie de casos de QCE por ADV iniciados en una consulta oftalmológica en Santiago. 


\section{Pacientes y Métodos}

El estudio fue aprobado por el Comité de Ética del Hospital Clínico de la Universidad de Chile y se adecuó a la declaración de Helsinki. Se realizó después de obtener el consentimiento informado de cada paciente.

Pacientes: El estudio se realizó entre los meses de abril y agosto del año 2006. El caso índice de la serie de casos de queratoconjuntivitis aguda epidémica, correspondió a una paciente que acudió a la consulta oftalmológica del Hospital Clínico de la Universidad de Chile. Se incluyó en el estudio a todos sus contactos infectados sintomáticos. A todos los pacientes se les preguntó dirigidamente si habían estado antes cerca de otras personas con conjuntivitis. Se contactó telefónicamente a las personas que consultaron el mismo día que el caso índice y se les preguntó si habían presentado sintomatología oftalmológica sugerente de conjuntivitis. A cada participante del estudio se le realizó un examen oftalmológico acabado, se completó una ficha con los datos del paciente y se tomó registro fotográfico de las lesiones corneales.

Muestras: En cada caso se obtuvo secreción conjuntival con tórulas de dacrón estériles desde los fondos de saco conjuntivales. No se evaluó la presencia de virus en el instrumental utilizado en la sala de examen.

Detección de $A D V$ : Las muestras fueron enviadas en hielo al Programa de Virología del Instituto de Ciencias Biomédicas (ICBM) de la Universidad de Chile, donde se realizó aislamiento viral en cultivo celular, detección de antígenos virales por inmunofluorescencia indirecta y amplificación del genoma viral por reacción en de polimerasa en cadena (RPC) de acuerdo a procedimientos habituales del laboratorio ${ }^{21}$. Para la pesquisa de ADV se utilizaron partidores para amplificar un fragmento del gen del hexón de todos los ADV (RPC genérica) y para definir el género, las muestras positivas se sometieron a RCP con partidores específicos para cada uno de ellos, de acuerdo al protocolo de $\mathrm{Xu}$ y cols. ${ }^{21}$.

\section{Resultados}

La cadena de infección en el grupo de pacientes analizados se muestra en la Figura 1. Ella se habría iniciado a partir de una paciente que se encontraba al cuidado de un neonato que cursaba con cuadro de bronconeumonía por ADV, establecido clínicamente y confirmado por inmuno-fluorescencia indirecta (IFI). La paciente habría iniciado la sintomatología cuatro días previos al momento de consultar. Presentaba un cuadro característico de conjuntivitis folicular, con infiltrados sub-epiteliales y adenopatías dolorosas pre-auriculares. Fue examinada en ese momento por el Dr. 1, quién le mostró el caso a sus colegas, la Dra. 2 y el Dr. 3. Cinco días después el Dr. 1, la Dra. 2 y el Dr. 3 presentaron sintomatología compatible con QCE. Dos semanas después de que la Dra. 2 examinara a la paciente 1 , junto con su colega el Dr. 1, su madre y otra paciente examinada por ella en otro centro presentaron la misma sintomatología. Tres semanas después la hija de la Dra. 2 y la hija de la paciente 2 presentaron también el cuadro infeccioso.

\section{Examen oftalmológico de los afectados}

Un resumen de algunas de las características clínicas oftalmológicas se muestra en la Tabla 1. Todos los pacientes presentaron compromiso binocular asincrónico, conjuntivitis folicular, edema palpebral y adenopatías pre-auriculares sensibles. La duración del cuadro fue menor a cuatro semanas en todos los pacientes, excepto en la paciente 1 , el Dr. 1 y en las pacientes 2 y 3 , quienes además presentaron pseudomembranas e infiltrados sub-epiteliales.

La Figura 2 muestra el compromiso palpebral e hiperemia conjuntival de la Dra. 2. La Figura 3 muestra una foto de la paciente 2, quien presentaba infiltrados estromales bilaterales centrales, especialmente en el ojo izquierdo, así como una erosión corneal central en el mismo ojo. Dentro de los antecedentes oftalmológicos de la paciente 2 , destacaba que había sido sometida a cirugía refractiva

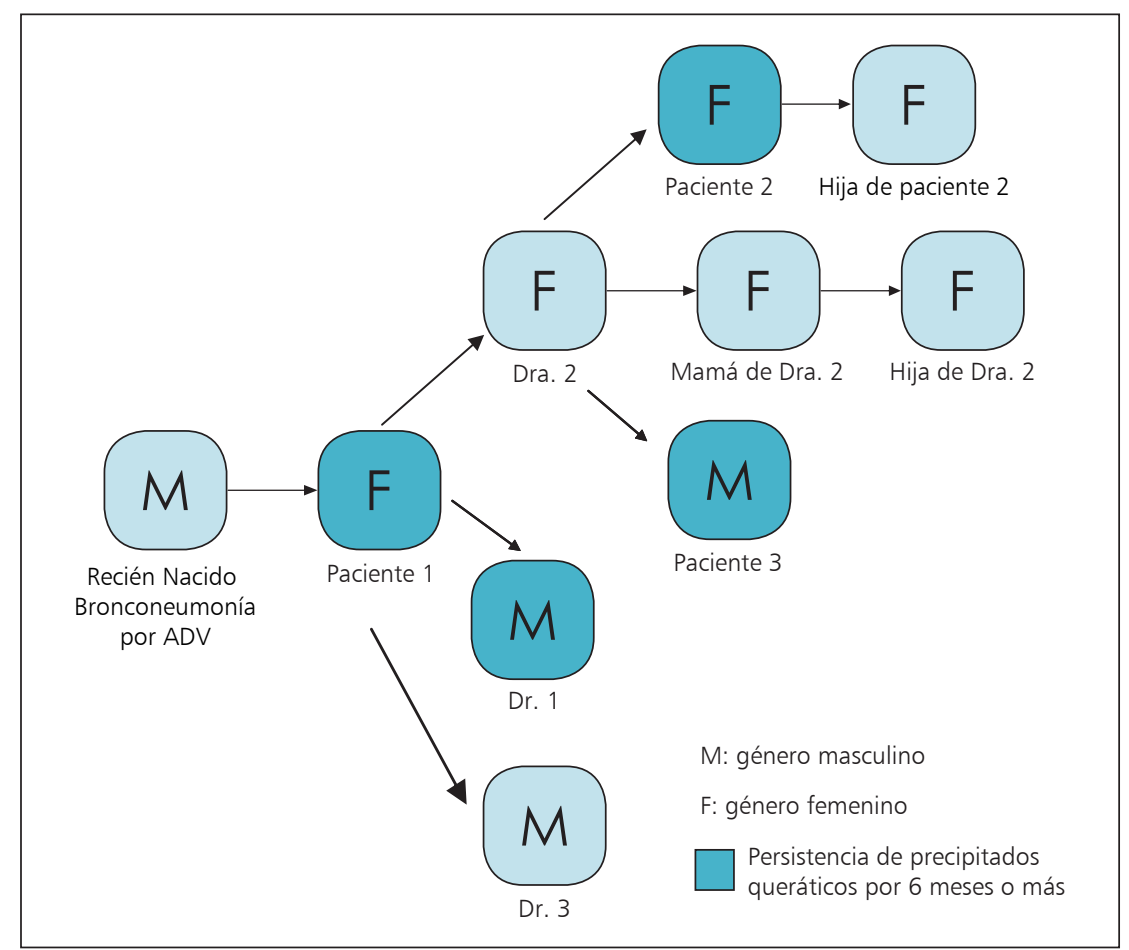

Figura 1. Cadena de infección de serie de casos de queratoconjuntivitis epidémica, Santiago, 2006. 
Figura 2. Hiperemia conjuntival intensa en Dra. 2.

\begin{tabular}{|c|c|c|c|c|c|c|}
\hline & $\begin{array}{c}\text { Conjuntivitis } \\
\text { folicular }\end{array}$ & $\begin{array}{c}\text { Edema } \\
\text { palpebral }\end{array}$ & $\begin{array}{l}\text { Adenopatía } \\
\text { pre-auricular } \\
\text { sensible }\end{array}$ & $\begin{array}{c}\text { Pseudo- } \\
\text { membranas }\end{array}$ & $\begin{array}{l}\text { Duración de } \\
\text { sintomatología } \\
\text { mayor } \\
4 \text { semanas }\end{array}$ & $\begin{array}{l}\text { Infiltrados } \\
\text { sub-epiteliales } \\
\text { por más de } \\
6 \text { meses }\end{array}$ \\
\hline Paciente 1 & + & + & + & + & + & + \\
\hline Dr. 1 & + & + & + & + & + & + \\
\hline Dra. 2 & + & + & + & - & - & - \\
\hline Dr. 3 & + & + & + & - & - & - \\
\hline Paciente 2 & + & + & + & + & + & + \\
\hline Paciente 3 & + & + & + & + & + & + \\
\hline Madre de Dra. 2 & + & + & + & - & - & - \\
\hline Hija de paciente 2 & + & + & + & - & - & - \\
\hline Hija de Dra. 2 & + & + & + & - & - & - \\
\hline
\end{tabular}
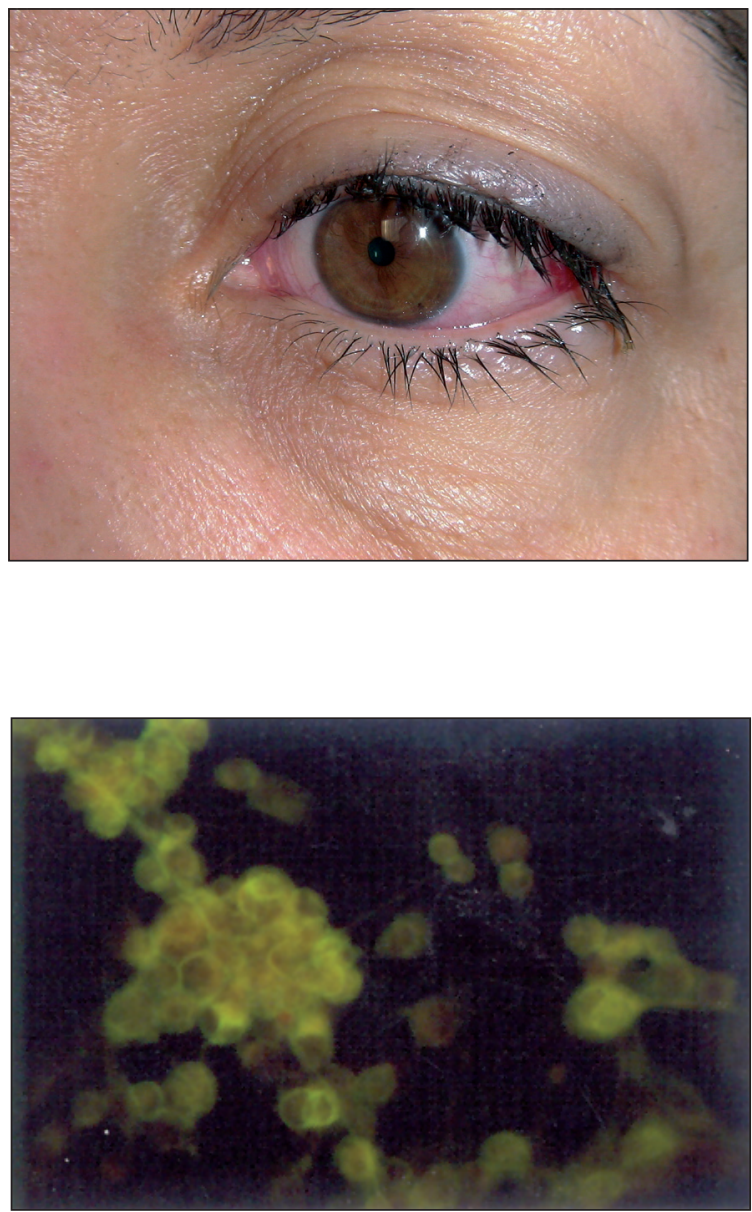

Figura 4. Confirmación del aislamiento viral mediante detección de antígenos. Inmunofluorescencia indirecta posterior a la inoculación de la muestra ocular infectada.

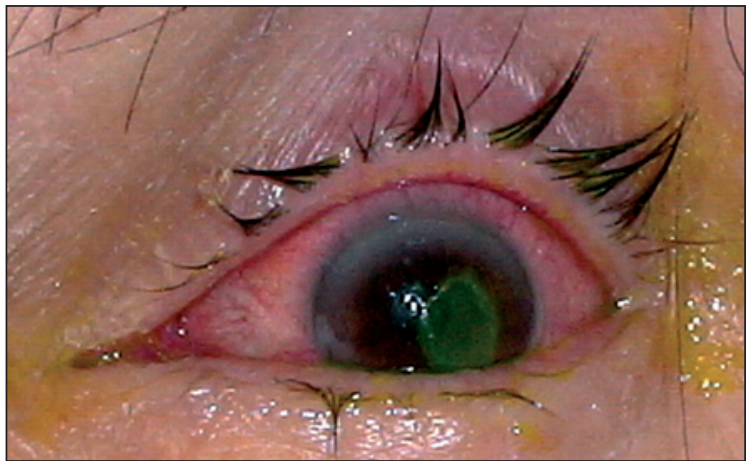

Figura 3. Hiperemia conjuntival intensa y defecto epitelial corneal para-central en paciente 2 .

Lasik* un año antes y estaba en tratamiento por conjuntivitis sicca grave. En los pacientes 2, 3, la paciente 1 y el Dr. 1, los infiltrados corneales persistieron por 8 meses.

La agudeza visual se mantuvo conservada en todos los pacientes, excepto en aquellos que persistieron con infiltrados sub-epiteliales por más de seis meses, quienes la recuperaron tras la desaparición de los infiltrados.

En todos los casos se realizó manejo sintomático con frío local, lubricación y en aquellos casos en que persistieron los infiltrados sub-epiteliales se administró colirio de corticoesteroides de bajo poder anti-inflamatorio como la fluorometalona de uso tópico.

* Nota del Editor: Lasik: Laser in situ keratomileusis (= esculpido de la córnea in situ). 


\begin{tabular}{|lcc|}
\hline \multicolumn{3}{|c|}{$\begin{array}{c}\text { Tabla 2. Aislamiento viral y determinación de } \\
\text { subgénero de ADV por RPC }\end{array}$} \\
\hline Cultivo celular & $\begin{array}{c}\text { Sub-género de } \\
\text { ADV detectado }\end{array}$ \\
\hline Paciente 1 & + & Género D \\
\hline Dr. 1 & + & Género D \\
\hline Dra. 2 & + & Género D \\
Dr. 3 & + & Género D \\
\hline Paciente 2 & + & Género D \\
\hline Paciente 3 & - & - \\
\hline Madre de Dra. 2 & + & Género D \\
Hija de paciente 2 & + & Género D \\
\hline Hija de Dra. 2 & - & - \\
\hline
\end{tabular}

Aislamiento y tipificación del ADV. En todos los pacientes, excepto la número 3 y la hija de la Dra. 2, se aisló ADV, con confirmación por IFI (Figura 4). La RPC definió que todos los ADV detectados fueron del género D (Tabla 2).

\section{Conclusiones}

La QCE con frecuencia es causada por ADV del subgénero D. Presenta una alta transmisibilidad y el período de incubación es de 8 a 12 días. El ADV es transmitido de persona a persona por distintos mecanismos, tales como fomites, inoculación directa en la conjuntiva a través de instrumentos como los tonómetros de contacto, dedos y soluciones. Dentro de las falencias de nuestro estudio debemos mencionar que no se buscó la presencia de adenovirus en el instrumental oftalmológico utilizado.

La evolución de la QCE sigue un patrón claramente establecido como la secuencia de queratitis epitelial superficial, queratitis epitelial profunda (día 8) e infiltrados subepiteliales (día 16). El cuadro clínico de queratoconjuntivitis dura 3 a 4 semanas y es autolimitado. Sin embargo, algunas cepas que frecuentemente producen cuadros de QCE autolimitadas, pueden evolucionar con complicaciones y persistencia de lesiones corneales por varias semanas, como se observó en cuatro pacientes de este estudio.

El tratamiento actual para la QCE es sintomático. No hay fármacos antivirales disponibles actualmente para el tratamiento de infecciones por ADV. Por lo tanto, la prevención es la principal medida de tratamiento.

Es importante considerar al médico como un potencial vector en la transmisión del virus. En base a la descrip- ción de esta serie de casos de QCE con participación de médicos, se enfatiza la necesidad de aplicar estrategias de prevención después de examinar a cualquier paciente, y especialmente en aquellos con sospecha de infección por ADV.

Existen medidas de prevención estándares como son el lavado de manos después del examen de cada paciente. Para examinar a pacientes con sospecha de infección por ADV se recomienda el uso de guantes o el uso de cotones de algodón para manipular los párpados, en lugar de hacerlo con los dedos. Para evitar el contagio a través de las gotitas a menos de un metro se recomienda que el paciente use mascarilla durante el examen oftalmológico en la lámpara de hendidura.

La desinfección del instrumental como lentes de prueba, tonómetros de contacto y elementos del microscopio en contacto directo con el paciente, como manillas, mentonera, cintillo frontal, mesa de la lámpara de hendidura y binoculares, con gluconato de clorhexidina o isopropil alcohol al 70\%, también han demostrado ser una medida efectiva $^{22}$. Así mismo debe tenerse especial cuidado con el uso de los dispensadores de gotas, los cuales no deben ponerse en contacto directo con el paciente.

Por otro lado, es fundamental la educación del paciente afectado, poniendo énfasis en las medidas de higiene como el lavado de manos frecuente y el uso de toallas desechables para limpiar secreciones en la piel.

\section{Resumen}

Introducción: La patología ocular infecciosa es frecuente en la consulta oftalmológica, especialmente la conjuntivitis y queratoconjuntivitis epidémica (QCE). Brotes de esta patología son causados por adenovirus (ADV) en el extranjero; en Chile se desconoce su impacto y características. Objetivos: Describir una serie de casos de queratonconjuntivitis epidémica por adenovirus. Material y Pacientes: Al caso índice y los contactos de una serie de casos de QCE por ADV que consultaron en el Hospital Clínico de la Universidad de Chile, entre abril y agosto de 2006, se les realizó examen oftalmológico completo y estudio de ADV por aislamiento viral, detección de antígenos y de genoma viral. Se estableció el género de ADV mediante reacción de polimerasa en cadena. Resultados: Los 9 pacientes infectados presentaron QCE característica. En algunos casos se observaron infiltrados sub-epiteliales corneales que se extendieron por más de seis meses. Tres pacientes eran médicos oftalmólogos. En 7 de los 9 pacientes examinados se aisló ADV; todos del género D. Conclusiones: En Chile, la QCE puede ser causada por el subgénero tipo D. El médico oftalmólogo es un potencial vector en la transmisión de ADV en un brote de QCE, por lo que es fundamental que sea considerado en las estrategias de prevención de esta patología. 


\section{Referencias}

1.- O'Donell B, Mc Cruden A B, Desselberger U. Molecular epidemiology of adenovirus in Glasgow 1981-1991. Eye 1993; 8-14.

2.- Schrauder A, Altmann D, Laude G, Claus H, Wegner K, Köhler R, et al. Epidemic conjunctivitis in Germany, 2004. Euro Surveill 2006; 11 (7): 185-7.

3.- Stefkovicová M, Sokolik J, Vicianová V, Madar R. Outbreaks of epidemic kerato-conjunctivitis in two hospital wards. Cent Eur J Public Health 2005; 13 (1): 29-31.

4.- Hendley J O. Epidemic keratoconjunctivitis and hand washing. N Engl J Med 1973; 289: 1368-9.

5.- Domínguez-Berjón M F, Hernando-Briongos P, Miguel-Arroyo P J, Echevarría J E, Casas I. Adenovirus transmission in a nursing home: analysis of an epidemic outbreak of keratoconjunctivitis. Gerontology 2007; 53 (5): 250-4. Epub 2007 Apr 11.

6.- Keenlyside R A, Hierholzer J C, D’Angelo L J. Keratocojunctivitis associated with adenovirus type 37: An extended outbreak in an ophthalmologist's office. J Infect Dis 1983; 147 (2): 191-8.

7.- Meléndez C P, Florentino M M, Martínez I L, López H M. Outbreak of epidemic keratoconjunctivitis caused by adenovirus in medical residents. Mol Vis 2009; 15: 557-62. Epub 2009 Mar 20.

8.- Asencio-Durán M, Romero-Martín R, García-Martínez J R, Peralta-Calvo J M,
Pérez-Blanco V, García-Caballero J, et al. Nosocomial outbreak of epidemic keratoconjuntivitis in a neonatal intensive care unit. Arch Soc Esp Oftalmol 2007; 82 (2): 73-9.

9.- Darougar S, Grey R H, Thaker U, Mc Swiggan A. Clinical and epidemiological features of adenovirus keratoconjunctivitis in London. Br Med J 1983; 67: 1-7.

10.- Hamada N, Gotoh K, Hara K, Iwahashi J, Imamura Y, Nakamura S, et al. Nosocomial outbreak of epidemic keratoconjunctivitis accompanying environmental contamination with adenoviruses. J Hosp Infect 2008; 68 (3): 262-8. Epub 2008 Mar 4.

11.- Horwitz M S. Adenoviruses. En: Fields BN, Knipe DM (eds). Virology. ( $2^{\mathrm{a}}$ ed). Raven Press, New York 1990, pp 1729-40.

12.- Wadell G, Allard A, Hierholzer J C. Adenoviruses. En: Murray PR, Baron EJ, Pfaller MA, Tenover FC, Yolken RH (eds). Manual of Clinical Microbiology, $9^{\text {th }}$ edition, 2007. American Society for Microbiology. Washington DC 2007, pp 970-7.

13.- Bernaola G, Luque W. Fisiopatología de las infecciones por adenovirus, Asociación de Médicos Residentes del Instituto de Salud del Niño, 2001 Oct - 2002 Marzo; 4 (2): 41-7.

14.- Zsengeller Z, Otake K, Hossain S A, Berclaz P Y, Trapnell BC. Internalization of adenovirus by alveolar macrophages initiates early proinflammatory signaling during acute respiratory tract infection. J Virology 2000; 74: 9655-67.

15.- White D O, Fenner F J. Adenoviruses. En:
White DO, Fenner FJ. Medical Virology 1994 $4^{\mathrm{a}}$ ed . Academic Press, San Diego, pp 306-17.

16.- West J, Rodman D M. Gene therapy for pulmonary diseases. Chest 2001; 119: 613-7.

17.- Cevenini R, Mazzarachio R, Rumplanest $F$, Donati M, Moroni A, Sambri V, et al. Prevalence of enteric adenoviruses from acute gastroenteritis. A five year study. Eur J Epidemiol 1997; 3: 147-50.

18.- Cruz J, Cáceres P, Cano F, Flores J, Bartlett A, Torún B. Adenoviruses types $40 \& 41$ and rotavirus associated with diarrea in children from Guatemala. J Clin Microbiol 1998; 28: 1780-8.

19.- Barnard D L, Dean Hart J C, Marmion V J, Clarke S K R. Outbreak in Bristol of conjunctivitis caused by adenovirus type 8 , and its epidemiology and control. Br Med J 1973; 2: 165-9.

20.- Larrañaga C, Martínez J, Palomino M A, Peña M, Carrión F, Avendaño L F. Molecular characterization of hospital-acquired adenovirus infantile respiratory infection in Chile using specie-specific PCR assays. J Clin Virol 2007; 39 (3): 175-81.

21.- Xu W, Mcdonough, Erdman D. Species-specific identification of human adenoviruses by a multiplex PCR assay. J Clin Microbiol 2000; 38 (11): 4114- 20

22.- Rutala W A, Peacock J E, Grgen M F, Sobsey M D, Weber D J. Efficacy of hospital germicides against adenovirus 8 , a common cause of epidemic keratoconjunctivitis in health care facilities. Antimicrob Agents Chemother 2006; 50: 1419-24. 